\title{
Los salarios en la economía política clásica: Algunas consideraciones sobre la doctrina clásica del mercado de trabajo
}

Emilio díaz CALleja*

\section{Introducción}

El interés por el pensamiento de los economistas clásicos, más allá del compromiso ineludible del historiador del análisis económico, ha encontrado nuevo estímulo en la situación de desconcierto que actualmente se vive en el ámbito de la teoría económica. En las últimas décadas, la llamativa acumulación de anomalías en la contraposición de las explicaciones teóricas convencionales con la evidencia empírica disponible ha llevado a muchos practicantes de esta disciplina a cuestionar seriamente tanto los fundamentos propios de la metodología dominante en economía (Katouzian, 1980), como los pilares básicos sobre los que se asienta la estructura lógica de la teoría económica desarrollada en el ámbito de la corriente principal del análisis económico (Weeks, 1989). Esta situación crítica es especialmente notoria en sectores particulares de la teoría económica, como se ha puesto de manifiesto recientemente, entre otros, en los importantes trabajos de R. Wray (1990), en el campo de la teoría monetaria, de H. Botwinick (1993), en la teoría de los salarios, y de A. Shaikh $(1980,1990)$ en los dominios de la teoría de la competencia y el comercio internacional.

No se pretende en este trabajo, sin embargo, indagar en las insuficiencias y limitaciones de la estructura teórica desarrollada por la escuela hegemónica del análisis económico, ni por tanto en los subsecuentes intentos de superarla por parte de sus críticos. No obstante, será oportuno llamar la atención en este punto sobre una circunstancia notable; a saber, que buena parte de las teorías alternativas al análisis convencional se han desarrollado dentro

\footnotetext{
* Profesor del Departamento de Economía Aplicada II. Universidad de Sevilla.
} 
de una estructura lógica que, frente a la propia de la economía ortodoxa, hunde sus raíces en la economía política clásiča ${ }^{1}$.

La importancia de este resultado radica, desde luego, en las eventuales ventajas que puede suponer, en la investigación sistemática de los fenómenos económicos, adoptar el punto de vista de la economía clásica, que puede contribuir de forma decisiva a superar el callejón sin salida en el que se halla metida la teoría económica moderna (Guerrero, 1995). Esto supone no sólo reconocer que el punto de vista de economistas clásicos puede aportar mucho en la comprensión de los problemas económicos que padecen en la actualidad las sociedades capitalistas, sino que significa también que dicho punto de vista no se ha incorporado, más que de forma superficial, a la teoría económica dominante dentro del análisis económico moderno, por mucho que los economistas neoclásicos insistan en proclamarse herederos del pensamiento de los economistas clásicos, incluso en el caso - lo que no deja de resultar llamativo_ de la teoría de la distribución (Samuelson, 1978; Hollander, 1979).

En los siguientes epígrafes nuestra intención es desvelar ciertos aspectos de la estructura lógica de la economía política clásica que, en nuestro criterio, son enormemente relevantes para comprender el funcionamiento del mercado de trabajo, a pesar de que tales aspectos han sido olvidados o, en el mejor de los casos, relegados a un segundo plano por la economía convencional. No obstante, debe aclararse que el análisis aquí desarrollado es meramente una interpretación, y por cierto no la única posible, del punto de vista clásico. Esto es así fundamentalmente porque de lo que se trata es de aislar elementos singulares perteneciente a un sistema teórico que los economistas clásicos elaboraron con el fin de comprender el funcionamiento global de la economía de mercado. El campo problemático de este sistema teórico no es sólo vasto y complejo, sino que no se presta fácilmente al tipo moderno de análisis, en el que los modelos económicos se elaboran con un número limitado y explícito de proposiciones, definidas de forma inequívoca (Eltis, 1984; 311).

Por otra parte, dado que la interpretación aquí sugerida presenta evidentes diferencias con respecto a la interpretación dominante entre los economistas convencionales modernos, será útil considerar esta última interpretación como punto de referencia, cosa que nos permitirá no sólo dilucidar las diferencias teóricas entre los puntos de vista clásico y neoclásico sino, sobre todo, mostrar cómo el primero de estos puntos de vista puede ser utili-

${ }^{1}$ El ejemplo más ilustrativo en este sentido es, sin duda, el trabajo de Piero Sraffa Production of Comodities by Means of Comodities, en el que explícitamente se identifica el punto de vista térico con "el de los antiguos economistas clásicos desde Adam Smith a Ricardo", punto de vista que, en opinión de este autor, "ha sido sumergido y olvidado desde el advenimiento del método marginalista" (Sraffa, 1960; 11). 
zado para mejorar en forma sustancial nuestra comprensión de problemas económicos actuales.

\section{La teoría clásica de la distribución}

Los economistas modernos suelen creer, no sin cierta dosis de confusión, que los economistas clásicos, que sentaron los principios básicos de esta ciencia, elaboraron una teoría de la distribución similar en lo fundamental a la teoría neoclásica hoy dominante, aunque con ciertas inconsistencias lógicas derivadas de la adopción de una teoría del valor - a saber, la teoría laboral del valor - errónea, inconsistencias que fueron definitiva y felizmente superadas tras la denominada "revolución marginalista", en el último tercio del siglo pasado. Es decir, se trata en suma de la atribución a los clásicos de la idea central de que los factores de producción - tierra, trabajo y capital- reciben una remuneración que se corresponde, en general, con su contribución al proceso de producción: tal es la naturaleza auto-reguladora de la ley general de los mercados, esto es, la ley de la oferta y la deman$d a^{2}$.

En lo que se refiere específicamente a la teoría de los salarios, que desde el punto de vista convencional constituye simplemente el caso particular del "factor trabajo" dentro de la teoría de la distribución —esto es, de la "teoría de la formación de los precios de los factores", un apéndice a su vez de la teoría general de los precios-, la opinión dominante rechaza que los economistas clásicos considerasen los beneficios y la renta de la tierra como sustracciones o deducciones del producto del trabajo ${ }^{3}$, es decir, como formas particulares

${ }^{2}$ Esto equivale a decir, desde otro punto de vista, que entre los propietarios de dichos factores no pueden establecerse, con carácter general, relaciones de explotación, es decir, relaciones en las cuales el propietario de un factor dado (por ejemplo, capital) obtiene con carácter permanente una remuneración superior a su contribución a la producción social, esto es, un excedente económico, cuyo origen sólo puede ser la apropiación de una parte de la remuneración correspondiente a los propietarios de otros factores (por ejemplo, trabajo). Lo que es más, caso de producirse eventualmente "explotación", no hay razón alguna, en esta perspectiva, para esperar que sea un factor en particular el "explotado"; el capital puede ser explotado por el trabajo tanto como el trabajo puede serlo por el capital, pues "la explotación se define como la discrepancia entre la "remuneración" real del "factor" y una cierta remuneración ideal" (Shaikh, 1990; 33).

${ }^{3}$ Especialmente importante es el caso de Adam Smith, pues La riqueza de las naciones no sólo constituye una gran síntesis de las aportaciones realizadas por los economistas que le anteceden, particularmente los fisiócratas, sino que será el punto de referencia de las discusiones posteriores entre los economistas. En este sentido, Hollander $(1973,1987)$ es quizá el autor que más incapié ha puesto en rechazar la idea de que dentro del sistema smithiano sea necesario tratar los beneficios y la renta de la tierra como un excedente económico, insistiendo por el contrario en la importancia que concede Smith a la contribución de la tierra y el capital al proceso de 
de excedente económico ${ }^{4}$.

Adviértase que se trata, contra lo que pudiera parecer, de un punto clave en la explicación de la formación de los salarios y, en consecuencia, del propio funcionamiento del mercado agregado de trabajo. Para mostrarlo, bastará que pensemos en lo siguiente: al adoptar una teoría de los precios basada en la "aditividad" de los costes de producción, los economistas convencionales modernos aducen que el encarecimiento autónomo del precio relativo de un factor productivo - especialmente, el encarecimiento del trabajo, dada la importancia cuantitativa de los salarios en la estructura de costes de las empresas- provocará un encarecimiento de los productos, o inflación de costes, y, probablemente, una reducción del nivel de producción y empleo 5 .

Desde el punto de vista de la teoría clásica de los precios, de acuerdo con la interpretación aquí sugerida, basada en el principio regulador del valor-trabajo y el excedente económico, deben señalarse dos cosas: en primer lugar, que las variaciones del precio del trabajo no se conciben en general como algo autónomo, sino más bien como la resultante de la acción conjunta de las fuerzas económicas, sociales e institucionales características del sistema; en segundo lugar, que caso de producirse un incremento del precio del trabajo, el efecto fundamental no será en general la elevación del precio de los productos, sino la reducción del excedente económico — beneficio—, si todo lo demás no varía (Stirati, 1994; $185-186)^{6}$.

producción. Sin embargo, caben pocas dudas de que Smith no analiza la distribución del producto en términos de la contribución de tierra, trabajo y capital a la producción de riqueza, sino en base a la idea de que el salario es inferior al producto total, parte del cual queda así a disposición de los propietarios de la tierra y el capital. No obstante, no puede negarse que existe cierta confusión en la presentación de este autor de la teoría laboral del valor, y que hay algunos elementos en la obra de Smith que inducen a pensar en una teoría "aditiva" de costes producción en la formación de los precios, que fueron criticados por David Ricardo. Schumpeter, por su parte, que reduce la tarea de Smith a la de mero "codificador" de la teoría del valor de sus contemporáneos y predecesores, no encuentra razones suficientes para considerar que la teoría del valor de Smith constituya verdaderamente una teoría del coste de producción en el sentido moderno (Schumpeter, 1954; 356-360).

${ }^{4}$ La interpretación de los clásicos basada en el excedente económico se remonta a Marx (1862), y ha sido defendida, entre otros, por Sraffa (1951), Meek (1967), Dobb (1973), Pasinetti (1974) y, más recientemente, por Walsh y Gram (1980), Howard (1983) y Garegnani $(1984,1987)$.

${ }^{5}$ Así, como puede leerse en un manual de amplia difusión, el resultado inmediato a nivel macroeconómico del denominado shock de oferta es, desde este punto de vista, doblemente aciago, porque eleva inmediatamente el nivel de los precios y reduce la producción; a más largo plazo, por otra parte, la senda de ajuste al equilibrio de pleno empleo pasa por la reducción de los salarios (Dornbush y Fisher, 1984, 439-440).

${ }^{6}$ Nótese que, en el primer caso, la dinámica económica contractiva tiene como determinante la elevación de los salarios, mientras que en el segundo caso, es exactamente lo contrario, ya que las variaciones del nivel de salario dependen ante todo de la acumulación de capital, de manera que solo indirectamente (al afectar a los beneficios) un aumento de los salarios afecta a la propia acumulación, en el sentido de desacelerarla. De esta forma, 


\section{El empleo, los salarios y la 'ley de la oferta y la demanda'}

Uno de los ejes centrales de la moderna teoría de los salarios es la extensión del principio de la demanda al mercado de trabajo. El principio se apoya decisivamente, en el caso de la demanda de trabajo, en la existencia de una relación inversa entre el nivel de empleo y la tasa de salario en el mercado agregado de trabajo: conforme crece (decrece) el nivel de empleo, disminuye (aumenta) la tasa de salario. Nótese que el principio de la demanda es de una naturaleza estática, toda vez que la demanda expresa una relación entre dos magnitudes por unidad de tiempo: en cada momento del tiempo es posible identificar una gama completa de combinaciones eficientes entre empleo y salario, es decir, una curva de demanda de trabajo ${ }^{7}$. Pues bien, de acuerdo con el planteamiento de este ensayo, la pregunta básica es la siguiente: ¿es posible identificar, entre los economistas clásicos, una noción de demanda de trabajo asimilable a la noción convencional?

Antes de responder a esta pregunta será conveniente, sin embargo, detenernos por un momento en recordar la relevancia de la cuestión que tenemos entre manos. En este sentido, y simplificando mucho, bastará darse cuenta de lo siguiente: la actuación del principio de la demanda en el mercado agregado de trabajo garantiza que no existe ningún obstáculo desde el punto de vista de la producción capitalista para que la economía funcione empleando completamente todos sus recursos, incluyendo la fuerza de trabajo disponible, esto es, con pleno empleo, de manera que, en esencia, el desempleo no puede ser atribuido a la organización de la producción en este sistema económico, sino a factores externos a la misma. Con otras palabras, el principio convencional de la demanda de trabajo, que postula una relación inversa entre empleo y salarios, constituye el fundamento analítico de la noción misma de pleno empleo, porque cualquier nivel concebible de empleo es alcanzable por la empresas capitalistas si la tasa de salarios es la adecuada. Esto es válido tanto para los neoclásicos puros como para los economistas de orientación keynesiana ${ }^{8}$.

el precio corriente del trabajo presentará oscilaciones continuas en tomo a cierto "centro gravitacional", el precio natural del trabajo, en cuya fijación intervienen factores institucionales (normas sociales) y también la fuerza respectiva de los contendientes (trabajadores y capitalistas), que no viene determinado entonces por la "oferta" y "demanda" de trabajo en el sentido moderno (Stirati, 1994; 191-198).

${ }^{7}$ Esto es lo mismo que decir que cuando los economistas modernos hablan de la demanda de trabajo en el sentido estático están pensando en una curva de demanda, que relaciona inversamente el empleo y los salarios. Véase, para una fundamentación microeconómica de este proceder, Bilas (1971; cap. 11).

${ }^{8}$ El propio Keynes deja sentado inequívocamente este principio en su Teoría general: “...con una determinada organización, equipo y técnica, los salarios reales y el volumen de producción (y por consiguiente empleo) está relacionados en una sola forma, de tal manera que, en términos generales, un aumento de la ocupación sólo puede ocurrir acompañado de un descenso de la tasa de salarios reales... En un estado conocido de organización, equipo y técnica, el salario real que gana una unidad de trabajo tiene una correlación única (inversa) con el volumen de ocupación" (Keynes, 1936; 27). 
Esto no quiere decir, desde luego, que el desempleo constituya algo inexistente o inexplicable dentro de la estrùctura de la teoría macroeconómica moderna, sino simplemente que el mismo no puede ser atribuido a la naturaleza de la producción capitalista. Adviértase que, ante esta conclusión, las explicaciones modernas de este fenómeno han de identificarse en la esfera de la circulación o intercambio, no en la esfera de la producción; específicamente, queda excluida categóricamente la posibilidad de que el desempleo esté asociado al modo en que se organiza la producción social; es decir, queda excluida la posibilidad de que el desempleo constituya una característica endógena al propio sistema económico.

Señalaremos de antemano que, en nuestra interpretación de los economistas clásicos, la idea de una relación inversa entre el nivel de empleo y la tasa de salario carece, en general, de fundamento. Como ha afirmado recientemente Antonella Stirati en su excelente estudio sobre la teoría de los salarios en la economía clásica, "por 'demanda de trabajo' los autores clásicos entienden una simple cantidad"; en concreto, "el número de horas de trabajo o de trabajadores requeridos por los empresarios capitalistas" (1994; 5). Esto equivale a rechazar que los clásicos concibieran la demanda de trabajo como una relación funcional o "curva de demanda", que únicamente puede sostenerse en la medida en que los supuestos en que se fundamenta la aplicación del principio de la demanda al mercado agregado de trabajo se demuestren compatibles, en general, con el análisis económico de la escuela clásica.

La identificación en los economistas clásicos, y particularmente en Adam Smith y sus contemporáneos, de una teoría del fondo de salarios, es precisamente una forma de hacer compatible la presencia del principio de la demanda en la determinación clásica de la tasa de salario, toda vez que el autor de La riqueza de las naciones, al igual que Turgot, Necker y otros economistas de la época, defendió sin duda la idea de que la tasa de salario poseía una tendencia a equipararse a lo largo del tiempo a un cierto nivel de consumo de los trabajadores, definido como nivel de subsistencia. Nótese que la efectividad del principio de la demanda en el mercado de trabajo depende vitalmente de que la tasa real de salario pueda descender hasta el nivel requerido cuando el nivel de empleo aumenta. Sin embargo, en el sentido moderno, el nivel de equilibrio de la tasa real de salario (nivel que garantiza el pleno empleo) no está sujeto a restricción alguna: en ausencia de perturbaciones en el mercado de trabajo, el libre juego de la oferta y la demanda garantiza un nivel tal de la tasa real de salario para el que toda la fuerza de trabajo disponible es empleada. En el sentido clásico, la actuación del principio de la demanda debe ser compatible con la restricción adicional de que existe un cierto nivel ex ante de la tasa real de salario: se trata de la tasa natural de salario, que tiende secularmente a igualarse al nivel de subsistencia. 
Ahora bien, partiendo de la teoría del fondo de salarios, y suponiendo además un mecanismo de ajuste poblacional ante variaciones de la tasa de salario, instancias ambas atribuidas a la economía clásica ${ }^{9}$, puede garantizarse una senda de equilibrio de pleno empleo: (i) supuesto que existe un fondo fijo de capital disponible para la contratación de trabajadores, toda expansión (contracción) de la oferta de fuerza de trabajo - población trabajadora disponible - podrá ser absorbida por las empresas mediante la reducción (incremento) de la tasa de salario; (ii) caso de que la tasa de salario llegue a situarse por debajo (por encima) del nivel de subsistencia, la población trabajadora tenderá a disminuir (aumentar), desencadenando el efecto descrito en el punto anterior; (iii) la tasa de salario de equilibrio, o tasa natural de salario, es entonces el nivel de subsistencia, en el cual la población trabajadora es estable, garantizándose de esta forma (por imperativo de una "ley natural") que, por virtud del mecanismo descrito en los puntos anteriores, el nivel de empleo es en todo caso el nivel de pleno empleo ${ }^{10}$.

Esta interpretación, que restablece el principio de la demanda como pieza clave del proceso de determinación de los salarios, implica la presencia, entre los economistas clási-

${ }^{9}$ La atribución de una teoría del fondo de salario a Adam Smith puede encontrarse en Cannan (1893), Lowe (1975), Bowley (1976) y Blaug (1985), entre otros. La atribución a este mismo autor de un mecanismo de ajuste de la población ante variaciones de la tasa de salario puede encontrarse en Hollander (1973) y Samuelson (1978).

10 Naturalmente, en ausencia de un mecanismo de ajuste poblacional puede darse el caso de desempleo. Esta circunstancia sería equivalente a la de "rigidez salarial" en términos de la economía convencional moderna, que en todo caso proviene de la oferta de fuerza de trabajo. La importancia del mecanismo de ajuste poblacional en esta interpretación de la economía clásica puede ilustrarse en el trabajo de Skinner (1979), que ha interpretado la teoria de los salarios de Smith a la luz de la categorías modemas de "tasa de salario de equilibrio" y "pleno empleo" sin necesidad de recurrir a la noción de un fondo fijo de salarios: (i) los incrementos de la demanda de trabajo característicos del estado progresivo se traducen en incrementos de la tasa real de salario, que se situará así por encima del nivel de subsistencia - que es la tasa natural de salario, es decir, de acuerdo con Skinner, la tasa de salario de equilibrio-, lo que desencadena el incremento de la oferta de trabajo -población trabajadora-; (ii) el retroceso de la demanda de trabajo propio de la economía en declive origina una reducción de la tasa real de salario hasta situarse por debajo del nivel de subsistencia, lo que provoca la reducción de la oferta de trabajo; (iii) en el estado estacionario, que constituye el escenario de equilibrio a largo plazo, no se presentan variaciones en la demanda de trabajo, de manera que la población trabajadora se reproduce sin cambios, y la tasa real de salario coincide con la tasa real de salario de equilibrio (nivel de subsistencia) sin necesidad de ajuste poblacional alguno. Nótese que, dentro de cada uno de los tres estados de la economía -decadente, estacionario y progresivo-, las variaciones del nivel de empleo a lo largo del tiempo son equivalentes a desplazamientos de la "curva de demanda", de ahí que se traduzcan en aumentos de la tasa de salario, dada la oferta de trabajo; por el contrario, las variaciones de la población trabajadora inducidas por los cambios en la tasa de salario, que significan desplazamientos de la curva de oferta de trabajo, se traducen en variaciones del empleo a lo largo de la curva de demanda. De esta forma se garantiza el equilibrio a largo plazo con pleno empleo de la fuerza de trabajo disponible y tasa natural de salario igual al nivel de subsistencia (cfr. Skinner, 1979; 164 y ss.). 
cos, de las categorías de "salario de equilibrio" y "pleno empleo", gracias a la presencia de un mecanismo de ajuste poblacional. Sin embargo, como ya se ha comentado, esta interpretación sólo es sostenible si los presupuestos en que se basa pueden ser identificados dentro de la estructura lógica de la economía política clásica. Analicémoslo con algún detalle.

(i) La formulación precedente de la teoría del fondo salarial presupone, en su versión nominal, la teoría cuantitativa del dinero. En efecto, esta última teoría implica que el precio del trabajo se forma simplemente mediante la confrontación de una suma dada de dinero - el fondo nominal de salarios, en poder de los empresarios capitalistas - con una cantidad determinada (pero variable) de fuerza de trabajo. La cantidad de dinero disponible para contratar a los trabajadores requeridos, para unas condiciones técnicas dadas, es por esta razón el determinante de los salarios nominales. Adicionalmente, siempre que la producción se suponga constante a corto plazo ${ }^{11}$, esto significa además que la tasa real de salarios se reduce conforme aumenta la cantidad de fuerza de trabajo disponible, que es la clave de la aplicación del principio de la demanda al mercado agregado de trabajo ${ }^{12}$.

(ii) Definida en términos reales, la teoría del fondo de salario combinada con el ajuste poblacional adecuado supone que la flexibilidad de los salarios ante variaciones de la can-

${ }^{11}$ A largo plazo, si el incremento de la producción viene acompañado de un incremento proporcional de la cantidad de dinero, el aumento del fondo de salarios definido en términos nominales permitirá un cierto crecimiento de la oferta de fuerza de trabajo sin que el salario real varie. Obsérvese que en todo caso, de acuerdo con esta interpretación, el determinante del tamaño de la población es el fondo monetario de salario pre-establecido. En este sentido, debe aclararse que la idea central de la teoría del fondo de salario, es que una determinada cantidad de dinero, o una determinada cantidad de medios de consumo en el caso de un fondo real de salarios, fija en cada caso, a pesar de que el fondo pueda variar con el tiempo, el nivel de los salarios y el empleo. En realidad, como se deduce claramente de los Principles de J. S. Mill (1865), la teoría del fondo de salario solo implica flexibilidad salarial desde el lado de la demanda, dado que el fondo de salario (normalmente en términos reales) se supone pre-determinado con independencia de la tasa de salario, pero no implica en sí mismo, necesariamente, ausencia de desempleo -que puede darse si las condiciones de la oferta de trabajo lo consienten, como en el caso de las uniones de trabajadores organizados- ni invariabilidad a largo plazo de la tasa real de salario, que depende (suponiendo una oferta de trabajo constante) de la variaciones a lo largo del tiempo del fondo salarial.

12 Alternativamente, considerando fijo el fondo real de salarios, los aumentos (reducciones) en el nivel de la tasa nominal de salario provocarán un incremento (disminución) de los precios de las mercancías consumidas por los trabajadores de la magnitud suficiente para asegurar el "reparto" del fondo fijo de salarios entre los trabajadores que se ofrecen en el mercado, por lo que el salario real percibido por cada trabajador variará en razón inversa al nivel de empleo.

Ambos procesos son, por otra parte, abiertamente incompatibles con la teoría de los salarios de Adam Smith y sus contemporáneos. Para Smith, por ejemplo, la fijación del salario es, en primera instancía, el resultado de un contrato (cuya naturaleza es asimétrica en el sentido de que la parte propietaria del capital impone normalmente sus intereses) que, dentro de un marco institucional dado y para unas condiciones también dadas de mercado, depende fundamentalmente de la fortaleza relativa de las partes (cfr. Smith, 1776; esp. cap. viii). 
tidad demandada de trabajo es infinita, con lo que queda excluida por completo la posibilidad misma de desempleo a la tasa natural de salario. El salario natural (nivel de subsistencia) es en realidad, como consecuencia, un salario de "equilibrio" en el sentido moderno. Nótese que, implícita en esta argumentación, aquí aparece la idea central, propia de la economía convencional moderna, de que siempre es posible aumentar el empleo aunque no se incremente el output, esto es, en ausencia de acumulación de capital ${ }^{13}$.

(iii) La dificultad principal, pese a todo, al intentar conciliar la postulada relación inversa entre empleo y salarios con la economía política clásica se refiere a lo siguiente: la acción del principio de la demanda hace totalmente superflua la idea de un precio "natural" del trabajo o tasa natural de salario, toda vez que la "tasa de salario de equilibrio" resultante de la interacción de la oferta y la demanda puede vaciar el mercado agregado de trabajo ante cualquier cantidad de fuerza de trabajo en disposición de emplearse, siempre que los salarios sean lo sufientemente flexibles; el nivel agregado de salario no tiene por qué ser pre-determinado, por tanto, por un nivel "natural", que constituye lógicamente una magnitud ex'ante. Es aquí donde debe actuar inevitablemente el ajuste poblacional, en la forma de la "ley del bronce de los salarios", que desencadena los movimientos adecuados de la oferta de fuerza de trabajo ${ }^{14}$.

${ }^{13}$ Lo cual sencillamente implica la noción de "función de producción", que constituye la base natural más general de derivación del principio de la demanda. Es decir, es bastante que cantidades específicas de capital y trabajo, o cualesquiera factores variables de la producción en el lenguaje moderno, puedan combinarse en distintas proporciones y, sin embargo, dejar inalterado el output, lo que presupone una relación funcional entre ambos factores variables ajustable como consecuencia de los cambios en los precios relativos. Está fuera de duda, sin embargo, que "no existe una función de producción en virtud de la cual la substitución de capital por trabajo dependa del precio relativo de los factores en la Riqueza de la naciones" - there is not production function where the substitution of capital for labour depends on relative factor prices in The Wealth of Nations'- (Eltis, 1984; 91). El mismo argumento es válido también en el caso de Ricardo (cfr. Pasinetti, 1974; 18).

${ }^{14}$ Adviértase que nos referimos a desplazamientos de la "curva" oferta de fuerza de trabajo, de acuerdo con esta interpretación. No obstante, el punto central de la argumentación es que tales desplazamientos está inducidos por los cambios en la tasa real de salario, que a su vez vienen regulados por el fondo de salarios dado en cada caso, ya que no existe en esta perspectiva otro mecanismo capaz de regularla. La alternativa, lógicamente, sería reconocer que ese otro mecanismo existe, y es bien real: el desempleo. No obstante, es posible asumir que el nivel de vida de los trabajadores (un salario real dado) incide sobre los cambios en la población trabajadora sin necesidad de asumir al mismo tiempo que dicho nivel de vida viene regulado por un fondo de salarios determinado. Es este precisamente el caso de David Ricardo, que reprocha a Malthus la adhesión a esta falsa doctrina: "Me parece que el Sr. Malthus propende mucho a pensar que la población aumenta únicamente por medio de una provisión previa de alimentos -"que es el alimento el que crea su propia demanda"-, que los matrimonios se estimulan proveyendo primero el alimento, en vez de considerar que el progreso general de la población resulta afectado por el incremento del capital, la consiguiente demanda de trabajo y el alza de los salarios, y que la producción de alimentos no es sino el efecto de esa demanda" (Ricardo, 1821; 303). Adicionalmente, Ricardo imputa abiertamente al menor ritmo de crecimiento del capital frente a la población, es decir, al desempleo, la responsabilidad principal de la pobreza (cfr. Stirati, 1994; 120). 
Sin embargo, con la sóla excepción de Malthus (1798), no es compatible con la teoría económica de los clásicos - incluyendo, además de Smith, Ricardo y sus seguidores, también a Necker (1775), Turgot (1766), Steuart (1766) y Cantillon (1755) - afirmar que la cantidad demandada de trabajo es el resultado de un fondo pre-establecido, fijo en cada caso, de medios de subsistencia, ni tampoco la invariabilidad del salario natural en el nivel de subsistencia física ${ }^{15}$. Como ha afirmado Eltis, a propósito de Adam Smith, "esta sugerencia, por tanto, parece reflejar la influencia de las formas modernas de pensamiento en la interpretación de los economistas pasados" $(1984 ; 56)$.

De lo anterior se deduce que no es correcto atribuir a los economistas clásicos, con la excepción apuntada ${ }^{16}$, ni una teoría del fondo de salarios, ya sea en términos nominales o en términos reales, ni un mecanismo de ajuste poblacional en la forma de una "ley del bronce de los salarios". Esta conclusión es importante porque significa, en sentido inverso, que los economistas clásicos rechazaron la regulación del mercado de trabajo sobre la base de la "ley de la oferta y la demanda" tal y como es conocida en nuestros días. Esto no significa que la economía clásica no reconociera y utilizara en sus análisis las nociones de oferta y demanda de trabajo, pues fueron precisamente estos pensadores los que dieron la primera caracterización sistemática de la "ley de la oferta y la demanda"; significa sólo que la ley de la oferta y la demanda en el sentido clásico no es en absoluto lo mismo que en el sentido neoclásico moderno.

\section{Acumulación, desempleo y salarios}

En el epígrafe anterior hemos concluido que, de acuerdo con esta interpretación, la economía clásica rechazaba, en general, que la de la cantidad demandada de trabajo fuera una función decreciente del precio corriente del trabajo (principio de la demanda), y que la oferta corriente de trabajo tuviera una relación funcional positiva con respecto a ese mismo precio (principio de población), de tal naturaleza que, bajo condiciones muy generales, el precio normal del trabajo resultara una consecuencia de la interacción de ambas funciones.

${ }^{15}$ Marx atribuye claramente a Malthus la paternidad de la "ley del bronce de los salarios", normalmente asociada al nombre de F. Lassalle: "De la "ley del bronce del salario" no pertenece a Lassalle, como es sabido, más que la expresión "de bronce"... ¿Y cuál es ésta? Es, como ya señaló Lange, poco después de la muerte de Lassalle, la teoría de la población de Malthus" (Marx, 1875; 46).

${ }^{16}$ Es curioso observar, sin embargo, que el sistema de economía política de Robert Malthus no parece haber ejercido una influencia significativa ni entre sus contemporáneos ni, desde luego, en el ámbito de la economía neoclásica posterior. Únicamente J. M. Keynes, en un famoso ensayo escrito en 1933 ("Robert Malthus: The First of the Cambridge Economist"), en el que llega a glorificar al autor del Essay on the Principle of Population, se da cuenta de la eventual importancia del clérigo anglicano para la economía convencional modema: " $\mathrm{Si}$ Malthus y no Ricardo hubiera sido el tronco del que brotó la ciencia económica del siglo XIX, cuánto más sabio y rico sería hoy el mundo!" (Keynes, 1933; 37). 
Por el contrario, los precios corrientes del trabajo, que oscilan continuamente a lo largo del tiempo, eran considerados por los economistas clásicos, en cada momento del tiempo, simples datos empíricos, no variables económicas. La preocupación de estos autores no era, por esta razón, la investigación de los siempre cambiantes precios de mercado de la fuerza de trabajo, sino el precio "medio" con respecto al cual los precios de mercado se aproximan y alejan de forma continua, que ellos denominaron precio "natural" del trabajo.

En esta interpretación, el precio natural del trabajo o salario natural se configura como el "centro gravitacional" de los salarios de mercado; en consecuencia, el nivel y las variaciones del primero no pueden ser explicados por el nivel y la variaciones de los segundos, circunstancia esta última que, por el contrario, constituye la predicción más importante de la 'ley de la oferta y la demanda' en el sentido neoclásico moderno. Nótese que, al rechazar esta última explicación, el salario natural de los clásicos no puede ser concebido en absoluto como un "salario de equilibrio", pues esta última categoría es el resultado de aplicar un principio regulador que no pertenece en absoluto al sistema principal de economía política de los autores de esta época.

El resultado anterior es de gran importancia, no ya porque los economistas neoclásicos se equivocan al atribuir erróneamente a los clásicos su propia forma de razonar, sino porque el punto de vista de la economía política clásica puede ser utilizado para explicar fenómenos económicos que tienen una enorme trascendencia en la actualidad, y que no tienen sin embargo respuesta satisfactoria dentro de la economía convencional moderna. En este sentido, será útil enumerar una serie de consideraciones generales que, de acuerdo con nuestra interpretación, se derivan de aplicar la estructura lógica de la economía clásica al estudio del mercado agregado de trabajo:

(1) El salario natural o normal no viene determinado por la proporción que, en cada momento, existe entre la cantidad demandada de trabajo y el tamaño de la población trabajadora, es decir, por la "ley de la demanda y la oferta" de trabajo en el sentido clásico. Esto significa que el salario natural está determinado, entonces, por causas distintas a la "ley de la oferta y la demanda".

Tres son los factores generales que determinan, en este contexto, el salario natural (real): (i) el mayor o menor progreso de la capacidad productiva del trabajo ${ }^{17}$; (ii) la con-

${ }^{17}$ El progreso de la capacidad productiva del trabajo presenta. para los economistas clásicos, una relación directa con el nivel del salario natural (en términos reales) sencillamente porque tiende a abaratar las mercancías consumidas por los trabajadores, lo cual constituye la condición necesaria de toda elevación de la cantidad de medios de vida necesarios para la reproducción de la clase trabajadora. En este sentido, es interesante comprobar cómo Ricardo, defensor a ultranza de la acumulación de capital, se opone en sus recomendaciones políticas a todo lo que él entiende que obstaculiza el progreso de la producción, porque esta eventualidad va en contra de los intereses de todas las clases sociales, como en el caso de las leyes de pobres vigentes en su época (Ricardo, $1821 ; 81$ y ss.). 
frontación entre trabajadores y capitalistas en la defensa de sus intereses antagónicos ${ }^{18}$; (ii) el marco institucional, que está configurado por una serie de normas sociales que establecen límites a la conducta interesada de los agentes ${ }^{19}$.

Los economistas clásicos fueron no obstante pesimistas en relación a la posibilidad de incrementos ilimitados del nivel de vida de los trabajadores, aunque admitieron que este sería el caso si el progreso de la acumulación no presentara obstáculos importantes, como los rendimientos decrecientes en la producción de bienes primarios ${ }^{20}$.

${ }^{18}$ Smith se expresa en este punto con tal contundencia que merece la pena detenerse en presentar su punto de vista: "Los salarios del trabajo dependen generalmente, por doquier, del contrato concertado por lo común entre estas dos partes, y cuyos intereses difícilmente coinciden. El operario desea sacar lo más posible, y los patronos dar lo menos que puedan. Los obreros están siempre dispuestos a concertarse para elevar los salarios, y los patronos, para rebajarlos", aunque "no es difícil prever cuál de las dos partes saldrá gananciosa en la disputa, en la mayor parte de los casos, y podrá forzar a la otra a contentarse con sus términos." Según Smith, la parte triunfante será la de los patronos no sólo debido a las ventajas que las leyes les conceden (mientras prohiben las asociaciones de trabajadores), y a la menor resistencia de los trabajadores (debido a su menor capacidad para subsistir sin nuevos aprovisionamientos de medios de consumo), sino también a que los patronos "siempre y en todo lugar, mantuvieron una especie de concierto tácito, pero constante y uniforme, para no elevar los salarios por encima de su nivel actual". Finalmente, aunque por lo general los obreros se someten sin resistencia, a veces los trabajadores se coaligan para defenderse o elevar el precio de su trabajo, promoviendo alborotos; no obstante "los obreros pocas veces sacan fruto alguno de esas tumultuosas manifestaciones, las cuales ... fracasan generalmente, sin otro resultado que el castigo o la ruina de los dirigentes" (Smith, 1776; 65-66).

Por otra parte, tanto Turgot como Necker insisten en misma idea, aunque introduciendo con mayor especificidad otro elemento, también compartido por Smith; a saber, que la mayor debilidad negociadora de los trabajadores es acompañada y alimentada, por lo común, por la desproporción que normalmente se da entre la cantidad de trabajo disponible y de la demanda de los mismos, esto es, con otras palabras, por la situación normal de desempleo y subempleo en que viven los obreros (cfr. Stirati, 1994; 36 y ss.).

${ }^{19}$ Este factor fue especialmente valorado por Adam Smith, y en general, como en la mayor parte de sus opiniones sobre el funcionamiento del sistema, era compartido por el conjunto de los que venimos denominando economistas clásicos, tanto entre los autores que le anteceden como entre los posteriores. Es este marco institucional el que impide que el salario natural pueda caer por debajo del nivel de subsistencia biológica: "aun cuando en las disputas con los trabajadores gocen generalmente de ventaja los patronos, hay, no obstante, un cierto nivel por debajo del cual parece imposible que baje, a lo largo del tiempo, el salario corriente de las ocupaciones de inferior categoría. El hombre ha de vivir de su trabajo y los salarios han de ser, por lo menos, lo sufientemente elevados para mantenerlo" (Smith, 1776; 66). Según Smith, a este salario debe añadirse además, siguiendo expresamente a Cantillon, una cantidad adicional necesaria para el mantenimiento de la familia, para poder perpetuar así "la raza de esos trabajadores". La idea de un marco institucional basado en "convenciones sociales" es desarrollada por Smith en su Teoría de los sentimientos morales (1759), donde este autor se opone a la doctrina utilitarista. Por esta misma razón, Smith, como también Steuart (que distinguirá expresamente entre "necesidades físicas" y "necesidades políticas"), no identifica el "salario de subsistencia" con el mínimo físico o biológico de sustento.

${ }^{20}$ En particular, Ricardo sostenía que los rendimientos decrecientes en la producción de los productos primarios terminaría por agotar las posibilidades de una expansión sostenida del capital capaz de garantizar el crecimiento demográfico de las naciones capitalistas. Es decir, en su análisis final, la acumulación de capital sería frenada, ante todo, por obstáculos naturales, lo cual de ninguna manera significa que esta fuera la única fuente de contradicciones identificada por Ricardo en el curso de sus investigaciones (cfr. Ricardo, 1821; especialmente, caps. v y xxxi). 
Por otra parte, defendieron la idea clave de que no sólo las fuerzas económicas, sino también factores históricos, institucionales y culturales tienen una importancia singular tanto en la fijación del nivel de los salarios como en su evolución a lo largo del tiempo. Estos factores están totalmente ausentes en el núcleo duro de la teoría neoclásica de la formación del salario en condiciones de libre competencia.

(2) En consonancia con las conclusiones anteriores, los economistas clásicos concibieron los salarios corrientes o de mercado como datos reales, tratando de explicar no su nivel o dinámica - algo en sí mismo intrascendente-, sino sus desviaciones o discrepancias con respecto al salario natural. Encontraron que tales discrepancias eran continuas y que eran ocasionadas, como para cualquier artículo o mercancía, por las condiciones impuestas por la competencia, que era concebida esencialmente como un proceso dinámico en virtud del cual el capital fluye de una rama a otra de actividad buscando maximizar su rentabilidad, igualando así las cuotas de ganancia de todas sus partes. A lo largo del proceso, la proporción entre oferta y demanda cambia continuamente, de manera que los precios corrientes gravitan en torno a los precios naturales.

Sobre esta base, no constituye ningún misterio que los economistas clásicos concluyeran que el nivel de los salarios depende, en general, de la acumulación de capital, y que los incrementos en dicho nivel no necesariamente se traducen en incrementos de los precios de las mercancías, sino, más bien, en una re-distribución del producto del trabajo ${ }^{21}$. Ello contrasta con el punto de vista hoy dominante entre los economistas modernos, que ven en los incrementos de los salarios una de las causas más importantes tanto de la inflación como de la desaceleración de la producción y el empleo.

(3) El desempleo constituye, para los economistas clásicos, un fenómeno que acompaña normalmente el desarrollo de la producción capitalista. Si bien es incorrecto atribuir a los clásicos la idea de que los salarios reales están fijados por una inexorable ley natural (la 'ley del bronce de los salarios'), no es menos cierto que estos autores rechazaron la idea de un salario real indefinidamente flexible a la baja, única forma de introducir en el sistema de economía política por ellos elaborado la idea del "pleno empleo" como un corolario de la auto-regulación del sistema económico. Lo que es más, en la teoría salarial de la eco-

${ }^{21} \mathrm{Al}$ aplicar la teoría laboral del valor, el precio relativo de un producto cualesquiera con respecto a otro (siendo ambos bienes reproducibles) depende de la cantidad relativa de trabajo a ellos incorporado en su producción, que regula su valor de cambio. Los incrementos de la capacidad productiva del trabajo en la producción de un determinado bien reduce pues su precio -es decir, reduce su precio relativo con respecto a la mercancía-dinero en circulación, por ejemplo el oro-. Por consiguiente, siempre que el aumento de la productividad del trabajo se manifieste en una reducción del precio de los artículos que forman el fondo de consumo de los trabajadores -lo cual no siempre es una pauta general, entre otras cosas porque el aumento de la producción agrícola puede 
nomía clásica el desempleo (y el subempleo) constituye el mecanismo básico que ajusta los precios corrientes del trabajo a su precio natural ${ }^{22}$.

En nuestro criterio, los resultados que acabamos de mostrar son importantes porque, como anotábamos al principio de este ensayo, son de extrema utilidad para la comprensión de problemas vitales que aquejan a la sociedad actual. En este sentido, estamos convencidos de que "teorías cuyo desplazamiento ha sido un progreso pueden a su vez desalojar a aquellas por las cuales fueron marginadas ellas mismas, y tanto su primera desaparición cuanto su regreso pueden ser beneficiosos para esa cosa curiosa que es el conocimiento científico" (Schumpeter, 1954; 335).

${ }^{22}$ Por supuesto, no sólo se trata de que el proceso de producción capitalista lleve implícito el desempleo como característica típica, en el sentido de que cumple una función necesaria para la regulación del sistema, sino de que el origen del desempleo no se debe buscar fundamentalmente en la órbita de la circulación _en el mercado de trabajo_. Por el contrario, las causas del desempleo van a estar vinculadas, en la economía clásica, bien a las restricciones impuestas por la naturaleza, o bien a las restricciones identificables en el propio seno de la producción social. Específicamente en esta última línea se encuentra el controvertido ensayo "On machinery", que Ricardo añadió a la tercera edición de su obra maestra, en el que este autor revisa su propia doctrina al sostener que "estoy convencido ahora de que la sustitución del trabajo humano por la maquinaria es, a menu. do, muy perjudicial a los intereses de la clase trabajadora" (Ricardo, 1821; 289). 


\section{BIBLIOGRAFÍA}

BILAS, R. A. (1971): TeOría Microeconómica. AlianZA, MAdRid, 1984.

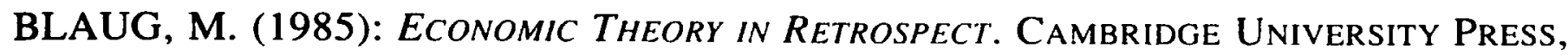
CAMBRIDGE.

BOTWINICK, H. (1993): PERSISTENT INEQUALITIES. WAGE DISPARITY UNDER CAPITALIST COMPETITION. PRINCETON UNIVERSITY PRESS, PRINCETON.

BOWLEY, M. (1976): STUdies IN THE History of ECONOMIC THEORY BEFoRE 1870. MACMILLAN, LONDRES.

CANNAN, E. (1893): A History of the THEORIES of PRODUCTION AND Distribution IN ENglish Political ECONOMY from 1776 to 1848. Percival \& Co., Londres.

DOBB, M. H. (1973): Theories of Value and Distribution since Adam Smith. CAMBridge UnIVERSitTy Press, CAMBRIDGe.

DORNBUSH, R.; FISHER, S. (1984): MaCRoEConomía [TERCERA EdICIÓN]. MCGRAWHILL, MADRID, 1985.

Eltis, W. (1984): The Classical Theory of EConomic Growth. Macmillan, Londres, 1992.

GAREGNANI, P. (1984): "VAlue and Distribution IN the Classical Economists and MARX”, EN OXFORD ECONOMICS PAPERS, VOL. XXXVI, PP. 291-325.

GAREGNANI, P. (1987): "Surplus Approach to Value and Distribution", en J. Eatwell, M. Milgate, P. Newman (Eds): The New Palgrave: a Dictionary of EconoMICS. MACMILLAN, LONDRES.

GUERRERO, D. (1995): CompetITIVIDAD: TEORIA Y POLITICA. ARIEL, BARCELONA.

HOLLANDER, S. (1973): THE ECONOMICS OF ADAM SMITH. HEINEMAN, LoNDRES.

HOLLANDER, S. (1979): THE ECONOMICS OF DAVID RICARDO. UNIVERSITY PRESS, TORONTO.

HOLLANDER, S. (1987): ClassiCAL ECONomics. BASIL BlaCKWELl, OXford.

HOWARD, M. C. (1983): Profits in ECONOMIC ThEORY. MACMILLAN, LONDRES.

KATOUZIAN, H. (1980): IDEOLOGía Y mÉTOdo EN ECONOMÍA. H. BluME, MADRID, 1982.

KEYNES, J. M. (1933): "RobERT MALTHUS (1766-1834): El PRIMER ECONOMISTA DE CAMBRIDGE”, en R. Malthus: Primer ensayo sobre onbi.aCión. Alianza, Madrid, 1970. 
KEYNES, J. M. (1936): TEORÍA GENERAL DE LA OCUPACIÓN, EL INTERÉS Y EL DINERO. FONDO de Cultura Económica, México.

LOWE, A. (1975): “AdAm SMITH's SySTEM OF EquILIBRIUM GROWTH”, EN A. S. SKINNER, T. WILSON (EDS): ESSAYS ON ADAM SMITH. ClARENDON, OXFORD.

MALTHUS, R. (1798): Primer ensayo sobre población. AlianZa, Madrid, 1970.

MARX, K. (1862): Teorías sobre la PLUSVALÍa. Fondo de Cultura EConómica, MéXico, 1984.

MARX, K. (1875): CRÍTICA DEL PROGRAMa DE GOTHA. RICARdo AguLleRA, MADRID, 1971.

MILL, J. S. (1865): Principles of Polttical EConomy, en J. M. Robson (ED): Colleted WORKS OF J. S. MILL. TORONTO UNIVERSITY PRESS, TORONTO.

MEEK, R. L. (1967): ECONOMICS AND IDEOLOGY AND OTHER ESSAYS: STUDIES IN THE DEVELOPMENT OF ECONOMIC THOUGHT. CHAPMAN AND HALL, LONDRES.

PASINETTI, L. (1974): GROWTH AND INCOME DISTRIBUTION. ESSAYS IN ECONOMIC THEORY. Cambridge University Press, Cambridge.

RICARDO, D. (1821): PRINCIPIOS DE ECONOMÍA POLITICA Y TRIBUTACIÓN. [EDICIÓN A CARGO DE P. SRAFFa]. Fondo de Cultura EConómica, MÉxico, 1973.

Samuelson, P. A. (1978): "The Canonical Classical Model of Political EcoNOMY", EN JOURNAL OF ECONOMIC LITERATURE. VOL. XVI, DIC.; PP. 1415-1435.

SCHUMPETER, J. A. (1954): Historia del aNÁlisIS ECONÓMICO. ARIEL, BARCELONA, 1994.

SHAIKH, A. (1980): "The LAWS OF INTERNATIONAl EXChANGE”, EN E. Nell (ED): Growth, Profits and Property. Essays in the Revival of Polttical Economy. OXford, PP. 204-235.

SHAIKH, A. (1990): VALOR, ACUMULACIÓN Y CRISIS. ENSAYOS DE ECONOMÍA POLÍTICA. TERCER MUNDO EDITORES, BOGOTÁ.

SKINNER, A. S. (1979): A System of Social SCIENCE. Papers Related to Adam Smith. CLARENDON, OXFORD.

SMITH, A. (1759): The Theory of Moral Sentiments. OXford University PREsS, OXFORD, 1976.

SMITH, A. (1776): INVESTIGACIÓN SOBRE LA NATURALEZA Y CAUSAS DE LA RIQUEZA DE LAS NACIONES. [Edición a CARGo de E. CanNan]. Fondo de Cultura Económica, México, 1992. 
SRAFFA (1951): "INTRODUCCION", EN D. RICARDO: PRINCIPIOS DE ECONOMIA POLITICA Y TRIbUtación. Fondo de Cultura ECONÓmiCa, MEXICO, 1973.

SRAFFA, P. (1960): PRODUCCIÓN DE MERCANC/A POR MEDIO DE MERCANCIAS. PRELUDIO A UNA crftica de la TEorla EConomica. Oikos-tau, Barcelona, 1983.

STIRATI, A. (1994): THE THEORY OF WAGES IN ClaASICAL ECONOMICS. A STUDY OF ADAM

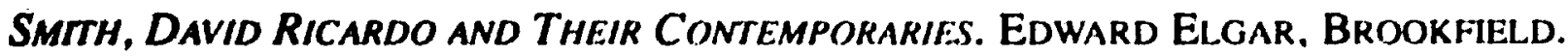

WALSH, V.; GRAM, H. (1980): ClassiCal and NEOCLASSICAL ThEORIES OF GENERAL EQUILIBRIUM. OXFORD UNIVERSITY PRESS, OXFORD.

WEEKS, J. (1989): A Critique of Neoclasical, Economics. St. Martin’s Press, New YORK.

WRAY, R. L. (1990): MONEY aND CREDTt IN CAPTIALIST ECONOMIES. THE ENDOGENOUS MONEY APPROACH. EDWARD ELGAR, BROOKFIELD. 